\title{
EGFR mutation testing and treatment decisions in patients progressing on first- or second-generation epidermal growth factor receptor tyrosine kinase inhibitors
}

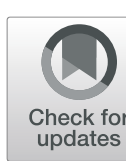

Anne C. Chiang ${ }^{1 *}\left(\mathbb{D}\right.$, Ancilla W. Fernandes², Melissa Pavilack², Jennifer W. Wu ${ }^{3}$, François Laliberté ${ }^{3}$, Mei Sheng Duh", Nabil Chehab ${ }^{2}$ and Janakiraman Subramanian ${ }^{5}$

\begin{abstract}
Background: The objective of this study was to investigate real-world EGFR mutation testing in patients with metastatic non-small cell lung cancer (NSCLC) upon progression on first-/second-generation epidermal growth factor receptor (EGFR)-tyrosine kinase inhibitors (TKI), and subsequent treatments received.

Methods: Flatiron Health electronic health records-derived database was used to identify adult patients with metastatic NSCLC treated with first-/second-generation EGFR-TKI from 11/2015-09/2017, with start of first EGFR-TKI defined as the index date. Patients were stratified by receipt of EGFR-TKI as first-line $(1 \mathrm{~L})$ or later-line $(2 \mathrm{~L}+)$ treatment. Mutation testing and subsequent therapies following first-/second-generation EGFR-TKI were described.

Results: Overall, 782 patients $(1 \mathrm{~L}=435 ; 2 \mathrm{~L}+=347$ ) were included. Median age was 69.0 years, $63.6 \%$ were female, $56.3 \%$ were white, $87.1 \%$ were treated in community-based practices, and $30.1 \%$ of patients died during the study period; median follow-up was 309.0 days. Among the $294(1 \mathrm{~L}=160 ; 2 \mathrm{~L}+=134)$ patients who received subsequent therapies, treatments included chemotherapy only $(1 \mathrm{~L}=15.6 \%$; $2 \mathrm{~L}+=21.6 \%)$, immunotherapy only $(1 \mathrm{~L}=13.8 \%$; $2 \mathrm{~L}+=41.0 \%)$, and targeted therapies $(1 \mathrm{~L}=70.0 \% ; 2 \mathrm{~L}+=36.6 \%)$. Specifically, 40 (25.0\%) $1 \mathrm{~L}$ patients and $7(5.2 \%)$ $2 \mathrm{~L}+$ patients received osimertinib as subsequent therapy. Before the start of subsequent therapy, EGFR T790M resistance mutation testing was performed in 88 (29.9\%) patients $(1 \mathrm{~L}=63$ [39.4\%]; $2 \mathrm{~L}+=25$ [18.7\%]). Of these patients, 25 (28.4\%) were T790M positive, among whom 24 (96.0\%) received osimertinib.

Conclusions: A third of patients received subsequent therapies on disease progression; only $30 \%$ of these were tested for EGFR-TKI resistance mutation, prior to receiving subsequent therapies. These results highlight the importance of choosing treatments in the $1 \mathrm{~L}$ setting that optimize benefits for patients with EGFRmutated NSCLC.
\end{abstract}

Keywords: NSCLC, EGFR-TKI, Real-world, EGFR mutation testing, Treatment patterns

\footnotetext{
* Correspondence: anne.chiang@yale.edu

'Yale School of Medicine, 20 York Street, New Haven, CT 06510, USA

Full list of author information is available at the end of the article
} 


\section{Background}

Systemic therapy for metastatic non-small cell lung cancer (NSCLC) varies according to tumor histology and mutation status of patients. Epidermal growth factor receptor (EGFR) mutation-positive tumors are highly responsive to EGFRtyrosine kinase inhibitor (TKI) therapy [1-5]. EGFR-TKIs provide improved clinical benefits, quality of life, and are considered more tolerable than chemotherapy [1-5]. Clinical guidelines recommend routine mutation testing and identification of EGFR mutations in all patients with NSCLC of non-squamous cell carcinoma (non-SCC) histology to identify who may benefit from approved EGFRTKIs [6-8]. However, most patients develop resistance to first- or second-generation EGFR-TKIs [9], with T790M, a resistance mutation in the EGFR gene, observed in approximately $50 \%$ of these patients $[10,11]$. EGFR T790M results in reduced binding of first- or second-generation EGFRTKIs, lessening EGFR-TKI-mediated inhibition and leading to disease progression [12, 13]. Identification of T790M prompted development of different therapeutic strategies to overcome resistance to EGFR-TKIs.

Osimertinib, a third-generation irreversible EGFR-TKI, selectively inhibits both EGFR-TKI sensitizing mutations and T790M. In November 2015, osimertinib received accelerated approval by the US Food and Drug Administration (FDA) to treat patients with EGFR T790M positive NSCLC whose disease progresses on or after prior treatment with EGFR-TKIs [14]. In April 2018, the FDA approved osimertinib as a first-line $(1 \mathrm{~L})$ treatment option for patients with EGFR exon 19 deletion (ex19del)/L858Rpositive metastatic NSCLC [15]. Furthermore, final overall survival results from the FLAURA study demonstrated significantly longer overall survival in those who received osimertinib versus comparator EGFR-TKI, with a $20 \%$ lower risk of death among patients with untreated advanced NSCLC with an EGFR-TKI sensitizing mutation [16]. Therefore, improved understanding of how EGFR mutation testing and treatment patterns have evolved in real-world practice among patients with metastatic NSCLC treated with first- or second-generation EGFRTKIs, since approval of osimertinib, is needed. Clinical guidelines recommend T790M testing for patients with resistance to first- or second-generation EGFR-TKIs, yet uptake of such testing in real-world practice is unknown [17]. In this real-world study, we aimed to describe the utilization of EGFR mutation testing of patients on progression with first- or second-generation EGFR-TKIs, subsequent treatments received, and the proportion of T790M positive patients treated with osimertinib.

\section{Methods}

\section{Data source}

This study used data from January 1, 2011 to September 30, 2017 from the Flatiron Health database, which is derived from electronic health records (EHR) containing longitudinal, patient-level data from a demographically and geographically diverse, nationally representative population in the US [18]. Additional data modules, including data on progression, site of metastases, and histology, were acquired for patients with metastatic NSCLC who received subsequent therapy after treatment with first- or second-generation EGFR-TKI to confirm disease progression. The data are de-identified, with provisions in place to prevent re-identification to protect patient confidentiality, in accordance with the 1996 Health Information Portability and Accountability Act.

\section{Study design and study population}

A retrospective longitudinal cohort study was conducted among adult patients with metastatic NSCLC treated with a first- or second-generation EGFR-TKI. To evaluate EGFR mutation testing and treatment patterns after first FDA approval of osimertinib (November 2015), patients were selected based on use of EGFR-TKI on or after November 1, 2015. Patients included had a diagnosis of lung cancer (International Classification of Diseases, 9th Revision, Clinical Modification diagnosis code: 162.x; International Classification of Diseases, 10th Revision, Clinical Modification diagnosis code: C34x, C39.9), at least two clinical visits on or after January 1, 2011, pathology consistent with NSCLC, and a diagnosis of stage IIIB-IV NSCLC or early-stage NSCLC and subsequent development of recurrent or progressive disease on or after January 1, 2011. Additionally, patients had to be aged 18 years or more at the time of their diagnosis, treated with a first- or second-generation EGFR-TKI (erlotinib, afatinib, or gefitinib) on or after November 1, 2015, have had at least one clinical visit within 3 months prior to EGFR-TKI initiation, and have initiated a firstor second-generation EGFR-TKI at least 6 months prior to end of data (September 30, 2017) to avoid right censoring. Patients with simultaneous use of multiple EGFR-TKI therapies were excluded. The index date was the date of initiation of EGFR-TKI treatment. The observation period extended from index date to study end date (date of death, end of clinical activity, or data cutoff).

\section{Study measures}

Demographic and clinical characteristic data collected included age at metastatic NSCLC diagnosis, sex, race, geographical region, physician practice type, insurance type, history of smoking, line of therapy for first- or second-generation EGFR-TKI, time from diagnosis (date of first recurrence or progressive disease after initial NSCLC diagnosis) to those who initiated EGFR-TKI in $1 \mathrm{~L}$ or second or later line $(2 \mathrm{~L}+)$, and Eastern Cooperative Oncology Group (ECOG) performance status. 
Treatment patterns were assessed and included subsequent therapies following first- or second-generation EGFR-TKI. NSCLC therapies were classified according to chemotherapies, immunotherapies, and targeted therapies (Table 1). Specifically, subsequent therapy was defined as a change in treatment after receiving a first- or second-generation EGFR-TKI. Patients who received the same EGFR-TKI agent in their index and subsequent therapy were considered to have continued on their initial EGFR-TKI therapy as subsequent therapy. Patients could have been classified into multiple therapy groups if receiving combination therapy (Table 2). Data on rates and results of EGFR mutation testing were reported for two time periods: 1) from metastatic NSCLC diagnosis to index date, 2) from after index date to date of initiation of subsequent therapy. For patients with multiple EGFR mutation tests, the test closest to index date was chosen for the baseline period, and the test closest to initiation of subsequent therapy was chosen for the observation period. In the database, EGFR and T790M were tested and reported together. Different types of mutations were also reported (i.e. T790M, ex19del, L858R point mutation in exon 2, other mutation types) for patients who were tested for EGFR and had positive results.
Patients with subsequent therapy following EGFR-TKI treatment were identified, and additional data abstraction was conducted to identify disease progression. Clinician documentation in medical charts, radiographic assessment, and/or pathology reports from the progression module in the database were reviewed to confirm whether disease progression occurred after EGFR-TKI initiation. Death of patients was determined from EHR and two external sources, including Social Security Death Index, and a commercial death dataset which collects information from obituaries, funeral homes and other sources to provide date of death within a week of death [19]. Patients without any clinical activity 3 months before data cutoff (i.e. July 1, 2017 to September 30, 2017) were considered lost to follow-up. Patients still treated with first EGFR-TKI therapy in the month prior to data cutoff were considered remaining on therapy. Patients not treated with first EGFR-TKI therapy in the month prior to data cutoff, but with evidence of clinical activity during the 3 months prior to data cutoff, were considered as having discontinued therapy.

\section{Statistical analyses}

Patient characteristics were described overall and separately for patients who received EGFR-TKI in the $1 \mathrm{~L}$ and

Table 1 Chemotherapies, immunotherapies, and targeted therapies for metastatic NSCLC

\begin{tabular}{|c|c|}
\hline Type of Therapy & Agents/Agent combinations \\
\hline \multicolumn{2}{|l|}{ Traditional Chemotherapies } \\
\hline Platinum monotherapy & Carboplatin, Cisplatin \\
\hline \multicolumn{2}{|l|}{ Platinum doublet therapy } \\
\hline With cisplatin & $\begin{array}{l}\text { Docetaxel, Etoposide, Gemcitabine, } \\
\text { Irinotecan, Paclitaxel, Pemetrexed, } \\
\text { Vinorelbine }\end{array}$ \\
\hline With carboplatin & $\begin{array}{l}\text { Docetaxel, Gemcitabine, Paclitaxel, } \\
\text { Pemetrexed, Vinorelbine }\end{array}$ \\
\hline Non-platinum based combination therapies & $\begin{array}{l}\text { Gemcitabine/Docetaxel, Gemcitabine/ } \\
\text { Paclitaxel, Gemcitabine/Vinorelbine, } \\
\text { Paclitaxel/Vinorelbine, Pemetrexed/ } \\
\text { Gemcitabine }\end{array}$ \\
\hline Maintenance therapy & Docetaxel, Gemcitabine, Pemetrexed \\
\hline Immunotherapies & Atezolizumab, Nivolumab, Pembrolizumab \\
\hline \multicolumn{2}{|l|}{ Targeted Therapies } \\
\hline ALK inhibitors & Alectinib, Brigatinib, Ceritinib, Crizotinib \\
\hline Angiogenesis inhibitors & Bevacizumab, Ramucirumab \\
\hline BRAF inhibitor & Vemurafenib \\
\hline CDK4/6 inhibitor & Palbociclib \\
\hline EGFR monoclonal antibody inhibitor & Cetuximab, Necitumumab \\
\hline EGFR tyrosine kinase inhibitors & Afatinib, Erlotinib, Gefitinib, Osimertinib \\
\hline MEK inhibitor & Trametinib \\
\hline Other monoclonal antibody inhibitors & Ipilimumab, Trastuzumab \\
\hline
\end{tabular}

ALK anaplastic lymphoma kinase, BRAF Raf isoform B, CDK cyclin dependent kinase, EGFR epidermal growth factor receptor, MEK mitogen activated protein kinase kinase 
Table 2 Treatment combinations among patients with a subsequent line of therapy

\begin{tabular}{|c|c|c|}
\hline Subsequent line of therapy & $\begin{array}{l}\text { Patients who received } 1 \\
\text { L EGFR-TKI and had } \\
\text { subsequent therapy } \\
(\boldsymbol{N}=160) \\
n(\%)\end{array}$ & $\begin{array}{l}\text { Patients who received } \\
2 \mathrm{~L}+\text { EGFR-TKI and had } \\
\text { subsequent therapy } \\
(\boldsymbol{N}=134), \\
n(\%)\end{array}$ \\
\hline Chemotherapy alone & 25 (15.6) & $29(21.6)$ \\
\hline Immunotherapy alone & $22(13.8)$ & $55(41.0)$ \\
\hline \multicolumn{3}{|l|}{ Targeted therapies } \\
\hline \multicolumn{3}{|l|}{ EGFR-TKI } \\
\hline Alone & $93(58.1)$ & $23(17.2)$ \\
\hline Plus chemotherapy & $3(1.9)$ & $3(2.2)$ \\
\hline Plus immunotherapy & $3(1.9)$ & $2(1.5)$ \\
\hline Plus other targeted therapies & $2(1.3)$ & $2(1.5)$ \\
\hline Plus chemotherapy, other targeted therapies & $1(0.6)$ & $3(2.2)$ \\
\hline \multicolumn{3}{|l|}{ Other targeted therapies } \\
\hline Alone & $4(2.5)$ & $2(1.5)$ \\
\hline Plus chemotherapy & $6(3.8)$ & $14(10.4)$ \\
\hline Clinical study drug ${ }^{\mathrm{a}}$ & $1(0.6)$ & $1(0.7)$ \\
\hline
\end{tabular}

$1 L$ first line, $2 L+$ second or later line, EGFR epidermal growth factor receptor, EGFR-TKI epidermal growth factor receptor-tyrosine kinase inhibitor

${ }^{a}$ Flatiron Health masks the names of clinical study drugs in the data due to the sensitive nature of patients in clinical trials

$2 \mathrm{~L}+$. Means (standard deviations) and medians were reported for continuous variables, and frequencies and proportions were reported for categorical variables. For patients who received EGFR-TKI in $1 \mathrm{~L}$ versus $2 \mathrm{~L}+$, results were compared using Wilcoxon rank sum test for continuous variables and chi-squares tests for categorical variables. The frequency and proportion of patients with EGFR mutation testing and specific results of testing from metastatic NSCLC diagnosis to index date were reported for the overall study population. Results of EGFR mutation testing between index date and subsequent therapy were reported for those who received subsequent therapy. The frequency and proportion of patients treated with specific types of subsequent therapies were described. The frequency and proportion of patients tested for EGFR (including T790M), testing results, and specific subsequent therapies received, were reported.

\section{Results}

\section{Patient characteristics}

The overall study population included 782 eligible patients with metastatic NSCLC using a first- or secondgeneration EGFR-TKI (shown in Fig. 1). In total, 435 patients received an EGFR-TKI as initial systemic therapy $(1 \mathrm{~L})$ and 347 as subsequent therapy $(2 \mathrm{~L}+)$. Of 435 patients who received EGFR-TKIs in $1 \mathrm{~L}$, only 160 had subsequent therapy. Of 347 patients who received EGFRTKIs in $2 \mathrm{~L}+, 134$ had subsequent therapy. Most patients on subsequent therapy were confirmed to have disease progression at chart review, while the remaining were not documented to have progressed. Of patients who received
EGFR-TKI in $1 \mathrm{~L}$ and $2 \mathrm{~L}+, 136 / 160(85.0 \%)$ and $115 / 134$ (85.8\%), respectively, had disease progression.

Overall, median age at diagnosis was 69.0 years, 497 (63.6\%) patients were female, $440(56.3 \%)$ were white, 681 (87.1\%) were treated in community-based practices, and $493(63.0 \%)$ had a history of smoking (Table 3). With regards to tumor histology, most $(83.8 \%)$ patients had nonSCC, $12.5 \%$ had SCC, and $3.7 \%$ had a tumor of unspecified histology. In total, 413 (52.8\%) patients had a documented ECOG assessment, among whom 315 (76.3\%) had an ECOG performance status of $0 / 1$. Overall median followup was 309.0 days. The median time from metastatic NSCLC diagnosis until date of EGFR-TKI initiation (index date) was 62 and 328 days for patients who initiated EGFRTKI in $1 \mathrm{~L}$ and $2 \mathrm{~L}+$, respectively. From diagnosis to index date, 555 (71.0\%) patients overall were tested for EGFR mutations. Among these patients tested, 355 (64.0\%) patients were EGFR mutation-positive and, of these, five (1.4\%) were T790M positive. Patients who received EGFR-TKI in $1 \mathrm{~L}$ were significantly older, more likely female, and less likely to be treated in community-based practices and have a history of smoking than patients who received EGFR-TKI in $2 \mathrm{~L}+$. Patients who received EGFR-TKI in $1 \mathrm{~L}$ were significantly less likely to undergo EGFR mutation testing from diagnosis to index date compared with those who received EGFR-TKI in $2 \mathrm{~L}+(292$ [67.1\%] versus 263 [75.8\%], $P=$ 0.008). However, among those who underwent EGFR mutation testing, patients who received EGFR-TKI in $1 \mathrm{~L}$ were more likely than patients who received EGFR-TKI in $2 \mathrm{~L}+$ to be EGFR mutation-positive (242 [82.9\%] versus 113 [43.0\%], $P<0.001)$. Notably, blood-based EGFR testing was 


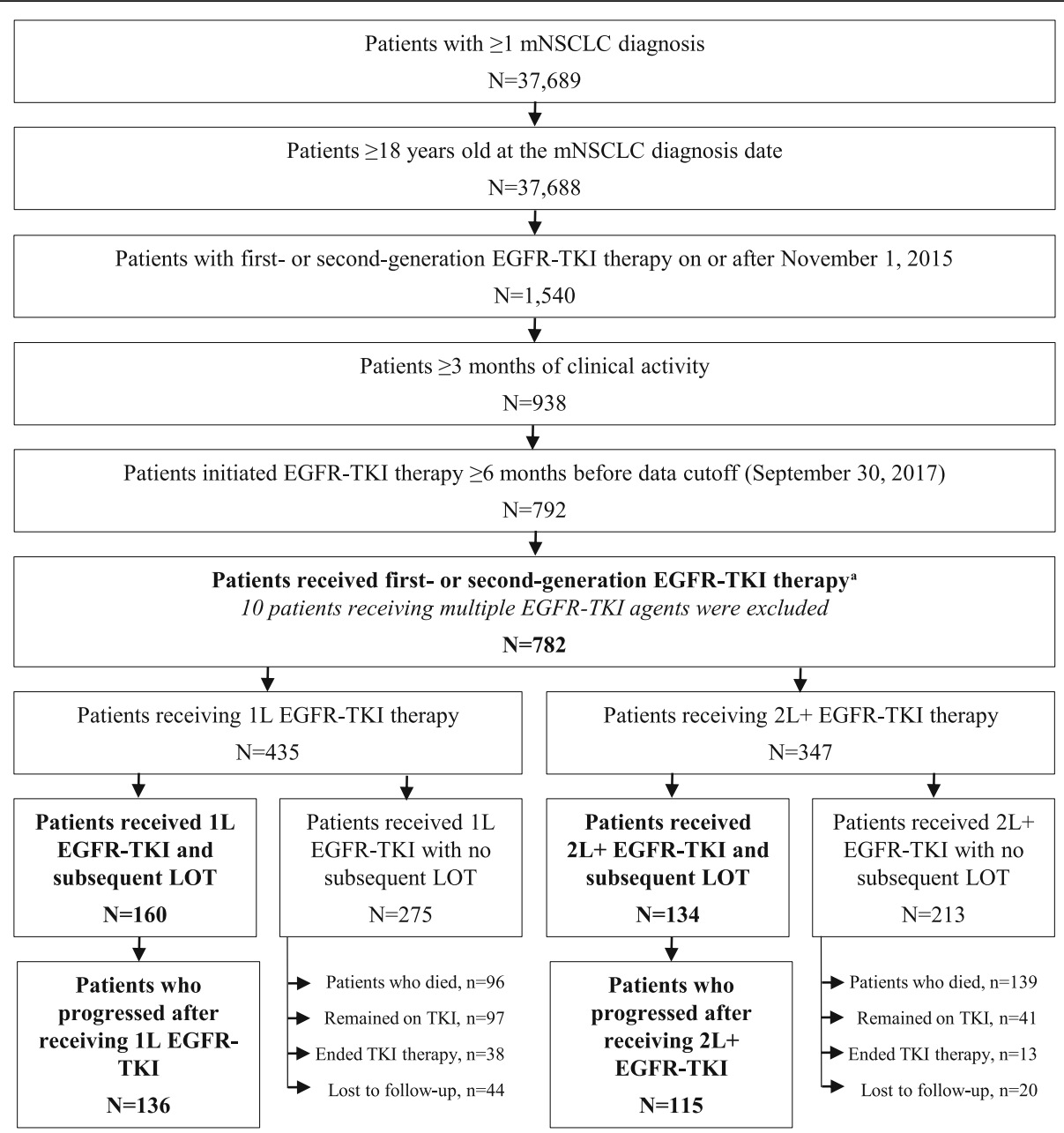

Fig. 1 Patient selection based on study inclusion/exclusion criteria. EGFR: epidermal growth factor receptor; LOT: line of therapy; NSCLC: nonsmall cell lung cancer; TKI: tyrosine kinase inhibitor. ${ }^{a}$ Patients receiving multiple EGFR-TKIs in the same line of therapy on or after November 1 , 2015 were excluded. This includes patients who started osimertinib plus a first- or second-generation EGFR-TKI or $\geq 2$ first- or second-generation EGFR-TKls.

lower in patients who received EGFR-TKI in $1 \mathrm{~L}$ and $2 \mathrm{~L}+$ (20 [6.8\%] versus 40 [15.2\%], $P=0.153)$.

\section{EGFR mutation testing and treatment patterns Patients who received subsequent therapy}

Of 782 patients with metastatic NSCLC who received EGFR-TKI in $1 \mathrm{~L}$ or $2 \mathrm{~L}+, 294(37.6 \%)$ had subsequent therapy, while the remainder did not (235 [30.1\%] died, 138 [17.6\%] remained on initial EGFR-TKI, 51 [6.5\%] discontinued EGFR-TKI, and 64 [8.2\%] were lost to follow-up; Fig. 2).

\section{Patients who received EGFR-TKI in $1 \mathrm{~L}$}

Of 160 (36.8\%) patients who had subsequent therapy, 25 (15.6\%) received chemotherapy only, 22 (13.8\%) immunotherapy only, and $112(70.0 \%)$ targeted therapies (Table 4); 99 (61.9\%) patients received targeted therapies only, three (1.9\%) targeted therapy plus immunotherapy, and 10 (6.2\%) targeted therapy plus chemotherapy (Table 2). More specifically, 40 (25.0\%) received osimertinib and $62(38.8 \%)$ continued on or switched to another EGFR-TKI (afatinib: 33 [20.6\%], erlotinib: 17 [10.6\%], gefitinib: 13 [8.1\%]; one patient received >1 EGFR-TKI) as subsequent therapy. Of the 160 patients, 9 (5.6\%) continued on initial EGFR-TKI plus added on chemotherapies, immunotherapies or other targeted therapies as subsequent therapy.

Of the 160 patients, $63(39.4 \%)$ were tested for T790M between index date and start of subsequent therapy (Fig. 3). Among the 63 patients, 23 (36.5\%) were T790M positive; of these, 22 (95.7\%) received osimertinib and one (4.3\%) received a clinical study drug. Among the 40 patients who were T790M negative, seven $(17.5 \%)$ received chemotherapy only, eight (20.0\%) immunotherapy only, and 25 (62.5\%) targeted therapies (afatinib: seven [17.5\%]; erlotinib: six 
Table 3 Demographic and clinical characteristics of patients who received EGFR-TKI

\begin{tabular}{|c|c|c|c|c|}
\hline & $\begin{array}{l}\text { Overall } \\
{[\mathrm{A}]}\end{array}$ & $\begin{array}{l}\text { Patients who received } \\
1 \mathrm{~L} \text { EGFR-TKl } \\
\text { [B] }\end{array}$ & $\begin{array}{l}\text { Patients who received } \\
2 \mathrm{~L}+\mathrm{EGFR}-\mathrm{TKI} \\
{[\mathrm{C}]}\end{array}$ & $\begin{array}{l}P \text {-value } \\
\text { for } \\
{[B] \text { vs [C] }}\end{array}$ \\
\hline & $(N=782)$ & $(N=435)$ & $(N=347)$ & \\
\hline Observation period, days, mean $\pm S D$ [median] & $371.5 \pm 312.3[309.0]$ & $435.5 \pm 329.7[372.0]$ & $291.3 \pm 268.7[220.0]$ & $<0.001$ \\
\hline $\begin{array}{l}\text { Age at metastatic NSCLC diagnosis, years, } \\
\text { mean } \pm \mathrm{SD} \text { [median] }\end{array}$ & $67.9 \pm 10.2[69.0]$ & $69.4 \pm 10.5[70.0]$ & $66.1 \pm 9.5[66.0]$ & $<0.001$ \\
\hline Female, $n(\%)$ & $497(63.6)$ & $315(72.4)$ & $182(52.4)$ & $<0.001$ \\
\hline \multicolumn{5}{|l|}{ Race, $n(\%)$} \\
\hline White & $440(56.3)$ & $246(56.6)$ & $194(55.9)$ & 0.857 \\
\hline Asian & $68(8.7)$ & $43(9.9)$ & $25(7.2)$ & 0.186 \\
\hline Black & $73(9.3)$ & $40(9.2)$ & $33(9.5)$ & 0.881 \\
\hline Other & $111(14.2)$ & $56(12.9)$ & $55(15.9)$ & 0.236 \\
\hline Unknown & $90(11.5)$ & $50(11.5)$ & $40(11.5)$ & 0.988 \\
\hline \multicolumn{5}{|l|}{ Geographic region, $n$ (\%) } \\
\hline Northeast & $142(18.2)$ & $81(18.6)$ & $61(17.6)$ & 0.707 \\
\hline West & $132(16.9)$ & 76 (17.5) & $56(16.1)$ & 0.621 \\
\hline Midwest & $111(14.2)$ & $51(11.7)$ & $60(17.3)$ & 0.027 \\
\hline South & $284(36.3)$ & $145(33.3)$ & $139(40.1)$ & 0.052 \\
\hline Unknown & $107(13.7)$ & 79 (18.2) & $28(8.1)$ & $<0.001$ \\
\hline Community-based practice, $n(\%)$ & $681(87.1)$ & $359(82.5)$ & $322(92.8)$ & $<0.001$ \\
\hline \multicolumn{5}{|l|}{ Insurance type, $n(\%)^{a, b}$} \\
\hline Commercial Health Plan & $379(48.5)$ & $198(45.5)$ & $181(52.2)$ & 0.065 \\
\hline Medicare & $300(38.4)$ & $169(38.9)$ & $131(37.8)$ & 0.754 \\
\hline Other payer & $246(31.5)$ & $127(29.2)$ & $119(34.3)$ & 0.127 \\
\hline Patient assistance program & $60(7.7)$ & $11(2.5)$ & $49(14.1)$ & $<0.001$ \\
\hline Medicaid & $47(6.0)$ & $17(3.9)$ & $30(8.6)$ & 0.006 \\
\hline Other government program & $31(4.0)$ & $11(2.5)$ & $20(5.8)$ & 0.021 \\
\hline Self pay & $7(0.9)$ & $4(0.9)$ & $3(0.9)$ & 0.935 \\
\hline Unknown & $115(14.7)$ & $73(16.8)$ & $42(12.1)$ & 0.066 \\
\hline \multicolumn{5}{|l|}{ History of smoking, $n$ (\%) } \\
\hline History of smoking & $493(63.0)$ & $234(53.8)$ & $259(74.6)$ & $<0.001$ \\
\hline No history of smoking & $287(36.7)$ & $199(45.7)$ & $88(25.4)$ & $<0.001$ \\
\hline Unknown & $2(0.3)$ & $2(0.5)$ & $0(0.0)$ & 0.206 \\
\hline \multicolumn{5}{|l|}{ Histology at NSCLC diagnosis, n (\%) } \\
\hline Non-squamous cell carcinoma & $655(83.8)$ & $402(92.4)$ & $253(72.9)$ & $<0.001$ \\
\hline Squamous cell carcinoma & $98(12.5)$ & $19(4.4)$ & $79(22.8)$ & $<0.001$ \\
\hline NSCLC histology - not otherwise specified & $29(3.7)$ & $14(3.2)$ & $15(4.3)$ & 0.417 \\
\hline \multicolumn{5}{|c|}{ Time from metastatic NSCLC diagnosis to index date, days, Q1 | Median | Q3 } \\
\hline Overall & 44 | 202 | 478 & $22|62| 336$ & $177|328| 553$ & $<0.001$ \\
\hline Stage $|-||| A$ & $29|158| 505$ & $17|50| 312$ & $171|372| 589$ & $<0.001$ \\
\hline Stage IIIB-IV & 72 | $221 \mid 472$ & $25|90| 337$ & $182|319| 552$ & $<0.001$ \\
\hline $\begin{array}{l}\text { ECOG assessment during the baseline period } \\
\text { (including index date), } n(\%)\end{array}$ & $413(52.8)$ & $212(48.7)$ & $201(57.9)$ & 0.011 \\
\hline \multicolumn{5}{|c|}{ ECOG performance status nearest or on the index date } \\
\hline 0 & $126(30.5)$ & $79(37.3)$ & $47(23.4)$ & 0.002 \\
\hline 1 & 189 (45.8) & 84 (39.6) & $105(52.2)$ & 0.010 \\
\hline
\end{tabular}


Table 3 Demographic and clinical characteristics of patients who received EGFR-TKI (Continued)

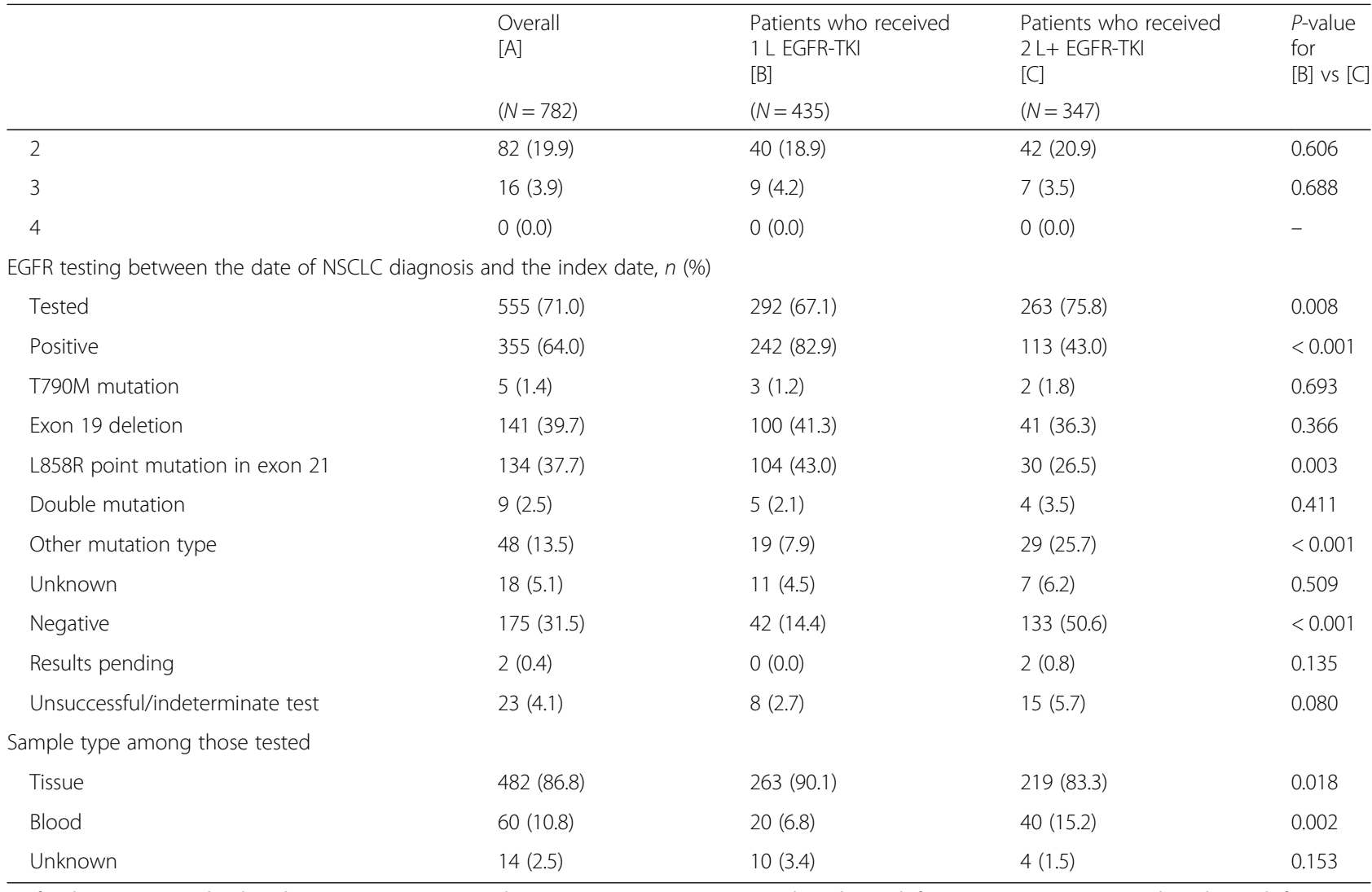

$1 L$ first line, $2 L+$ second or later line, ECOG European Oncology Cooperative Group, EGFR epidermal growth factor receptor, EGFR-TKI epidermal growth factor receptor-tyrosine kinase inhibitor, NSCLC non-small cell lung cancer, Q1 first quartile, Q3 third quartile; SD: standard deviation

a Evaluated on the index date

b Patients may have primary and secondary insurance (i.e. patients may have more than one type of insurance). Unknown insurance type was defined as patients without any types of insurance

[15.0\%]; osimertinib: five [12.5\%]; gefitinib: three [7.5\%]; other: four [10.0\%]). In the remaining 97 (60.6\%) patients not tested for EGFR mutations in the post-EGFR-TKI treatment period, 18 (18.6\%) received chemotherapy only, 14 (14.4\%) immunotherapy only, 65 (67.0\%) targeted therapies (afatinib: 26 [26.8\%]; erlotinib: 11 [11.3\%]; osimertinib: 13 [13.4\%]; gefitinib: 10 [10.3\%]; other: six [6.2\%]).

\section{Patients who received EGFR-TKI in 2 L+}

Of the 134 (38.6\%) patients with subsequent therapy, 29 (21.6\%) received chemotherapy only, 55 (41.0\%) immunotherapy (Table 4); 27 (20.1\%) received targeted therapies only, two (1.5\%) targeted therapy plus immunotherapy, and 20 (14.9\%) targeted therapy plus chemotherapy (Table 2). Specifically, seven (5.2\%) received osimertinib, and 27 (20.1\%) continued or switched to another EGFR-TKI (afatinib: 11 [8.2\%]; erlotinib: 13 [9.7\%]; gefitinib: five [3.7\%]) as subsequent therapy. Of the 134 patients, nine (6.7\%) continued on initial EGFR-TKI plus added on chemotherapies, immunotherapies or other targeted therapies as subsequent therapy.
Among the 134 patients, 25 (18.7\%) were tested for T790M (Fig. 4). Among the 25 patients tested, two (8.0\%) were T790M positive and both received osimertinib. Among the 23 T790M negative patients, six (26.1\%) received chemotherapy only, seven (30.4\%) immunotherapy only, and 10 (43.5\%) targeted therapies (afatinib: four [17.4\%]; osimertinib: two [8.7\%]; erlotinib: one [4.3\%]; other: three [13.0\%]). In the remaining 109 patients not tested for T790M in the post-EGFR-TKI treatment period, $23(21.1 \%)$ received chemotherapy only, 48 (44.0\%) immunotherapy only, and 37 (33.9\%) targeted therapies (erlotinib: 12 [11.0\%]; afatinib: seven [6.4\%]; gefitinib: five [4.6\%]; osimertinib: three [2.8\%]; other: 13 [11.9\%]).

Additional details on EGFR mutation testing between index date and subsequent therapy, and results are presented in Table 5.

\section{Discussion}

This study provided a novel opportunity to document treatment patterns, use of EGFR mutation testing, and how patients with metastatic NSCLC are managed in a real-world clinical setting based on data from EHR used 


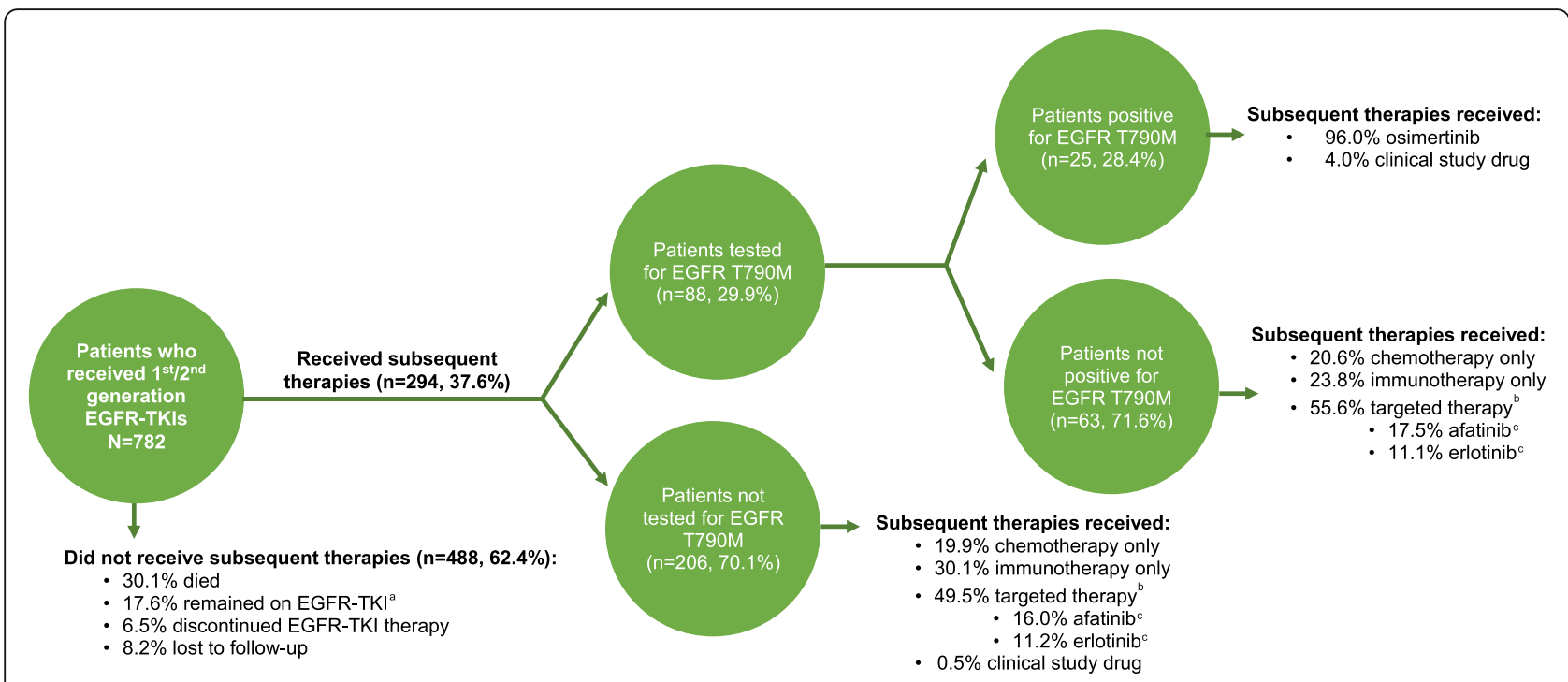

Fig. 2 Therapies received among all patients who received EGFR-TKI and were tested for EGFR T790M. EGFR: epidermal growth factor receptor; TKI: tyrosine kinase inhibitor. ${ }^{a}$ Patients still treated with their first EGFR-TKI therapy in the month prior to data cutoff were considered as remaining on EGFR-TKI therapy. ${ }^{\mathrm{b}}$ Targeted therapies included EGFR-TKIs, monoclonal antibody inhibitors, ALK inhibitors, and angiogenesis inhibitors. c The proportion of patients who received afatinib, erlotinib, and osimertinib are calculated over the total number of patients in the subgroup (e.g. the 206 patients for the analysis of patients not tested for EGFR T790M).

by US-based cancer care providers. These data illuminated several notable gaps in care. Despite clinical treatment guidelines recommending routine EGFR testing for all patients with metastatic NSCLC of non-SCC histology [6-8], here only $71 \%$ of newly diagnosed patients underwent EGFR testing prior to EGFR-TKI initiation. Consistent with this observation, another observational research study showed rates of documented EGFR testing rate to range between 41 and $97 \%$ in patients with newly-diagnosed metastatic NSCLC initiating systemic

Table 4 Treatment patterns among patients with metastatic NSCLC with a subsequent therapy

\begin{tabular}{|c|c|c|}
\hline \multirow[t]{2}{*}{ Subsequent therapy } & $\begin{array}{l}\text { Patients who received } 1 \mathrm{~L} \text { EGFR-TKI and had sub- } \\
\text { sequent therapy }\end{array}$ & $\begin{array}{l}\text { Patients who received } 2 \mathrm{~L}+\text { EGFR-TKI and had sub- } \\
\text { sequent therapy }\end{array}$ \\
\hline & $(N=160)$ & $(N=134)$ \\
\hline Chemotherapy alone, $n$ (\%) & $25(15.6)$ & $29(21.6)$ \\
\hline Immunotherapy alone, n (\%) & $22(13.8)$ & $55(41.0)$ \\
\hline Targeted therapies $n(\%)^{a}$ & $112(70.0)$ & 49 (36.6) \\
\hline EGFR-TKI ${ }^{b}$ & $102(63.8)$ & $33(24.6)$ \\
\hline $\begin{array}{l}\text { Continued on/switched to another 1st/2nd } \\
\text { gen. EGFR-TKI }\end{array}$ & $62(38.8)$ & $27(20.1)$ \\
\hline Afatinib & $33(20.6)$ & $11(8.2)$ \\
\hline Erlotinib & $17(10.6)$ & $13(9.7)$ \\
\hline Gefitinib & $13(8.1)$ & $5(3.7)$ \\
\hline Osimertinib ${ }^{c}$ & $40(25.0)$ & $7(5.2)$ \\
\hline Other targeted therapies ${ }^{d}$ & $10(6.3)$ & $16(11.9)$ \\
\hline Clinical study drug, $n(\%)$ & $1(0.6)$ & $1(0.7)$ \\
\hline
\end{tabular}

$1 L$ first line, $2 L+$ second or later line, $A L K$ anaplastic lymphoma kinase EGFR epidermal growth factor receptor, EGFR-TKI epidermal growth factor receptor-tyrosine kinase inhibitor, NSCLC non-small cell lung cancer

a Patients could have received targeted therapies alone or in combination with chemotherapy and/or immunotherapy

${ }^{b}$ Four patients had more than one EGFR-TKI as a subsequent therapy. Hence, the sum of the individual EGFR-TKIs (i.e. afatinib, erlotinib, geftinib, and osimertinib) will be greater than the total number who received EGFR-TKI

c Of the 47 patients who received osimertinib as subsequent therapies, 24 were tested T790M positive, seven were not positive for T790M, and 16 were not tested for EGFR mutations

${ }^{d}$ Other targeted therapeutic agents are listed in Table 1 


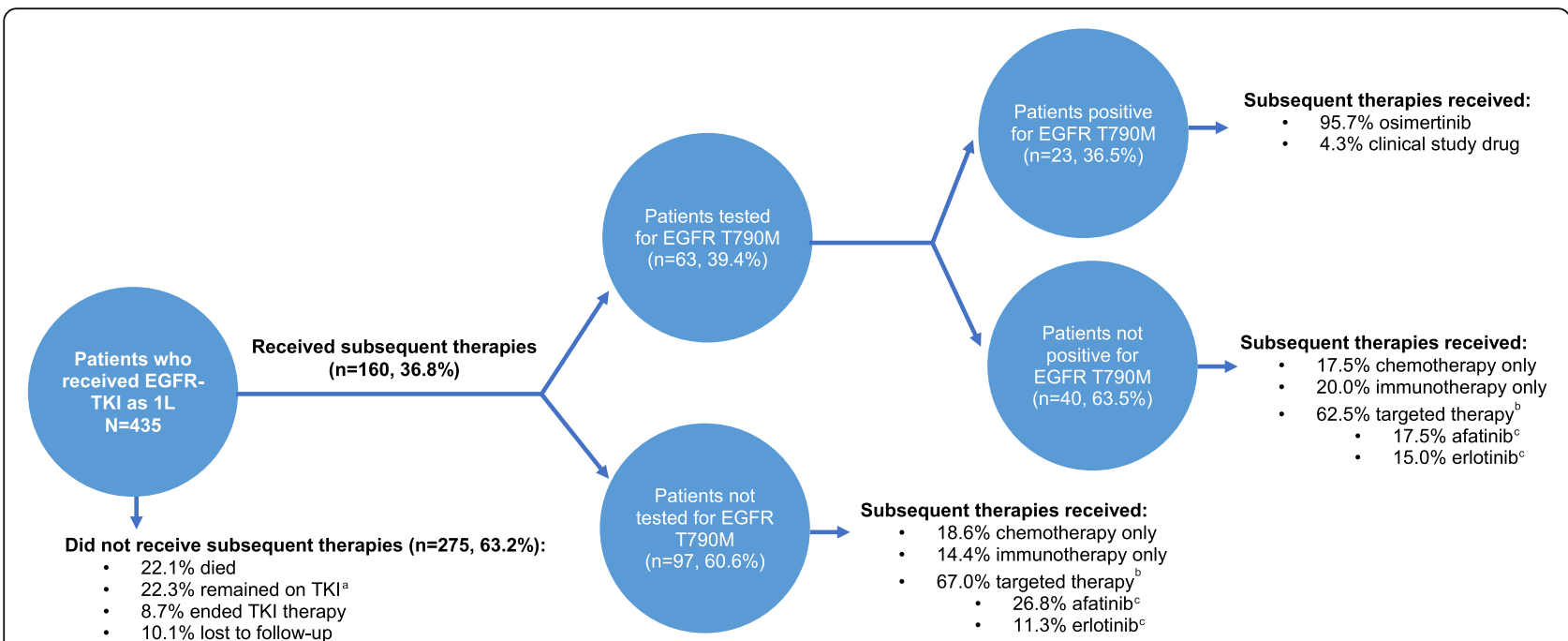

Fig. 3 Therapies received among patients who received EGFR-TKI in first line and were tested for EGFR T790M. 1 L: first line; EGFR: epidermal growth factor receptor; TKI: tyrosine kinase inhibitor. ${ }^{a}$ Patients still treated with their first EGFR-TKI therapy in the month prior to data cutoff were considered as remaining on EGFR-TKI therapy. ${ }^{\mathrm{b}}$ Targeted therapies included EGFR-TKIs, monoclonal antibody inhibitors, ALK inhibitors, and angiogenesis inhibitors. ${ }^{c}$ The proportion of patients who received afatinib, erlotinib and osimertinib are calculated over the total number of patients in the subgroup (e.g. the 97 patients for the analysis of patients not tested for EGFR T790M).

therapy (including EGFR-TKIs) [20]. Furthermore, the $30 \%$ rate of EGFR testing before subsequent therapy is consistent with previous estimates (30-48\%) [21-23] and emphasizes that, during the study period, a substantial proportion of patients initiated a subsequent therapy without updated information on the molecular profile of the tumor.
In this study, $87 \%$ of patients were treated in the community setting. Community-based practices may not be implementing standard testing guidelines or may have barriers related to EGFR mutation testing. Prior research has highlighted logistical challenges for communitybased oncologists regarding mutation testing, such as coordination of sample handling, long turnaround times,

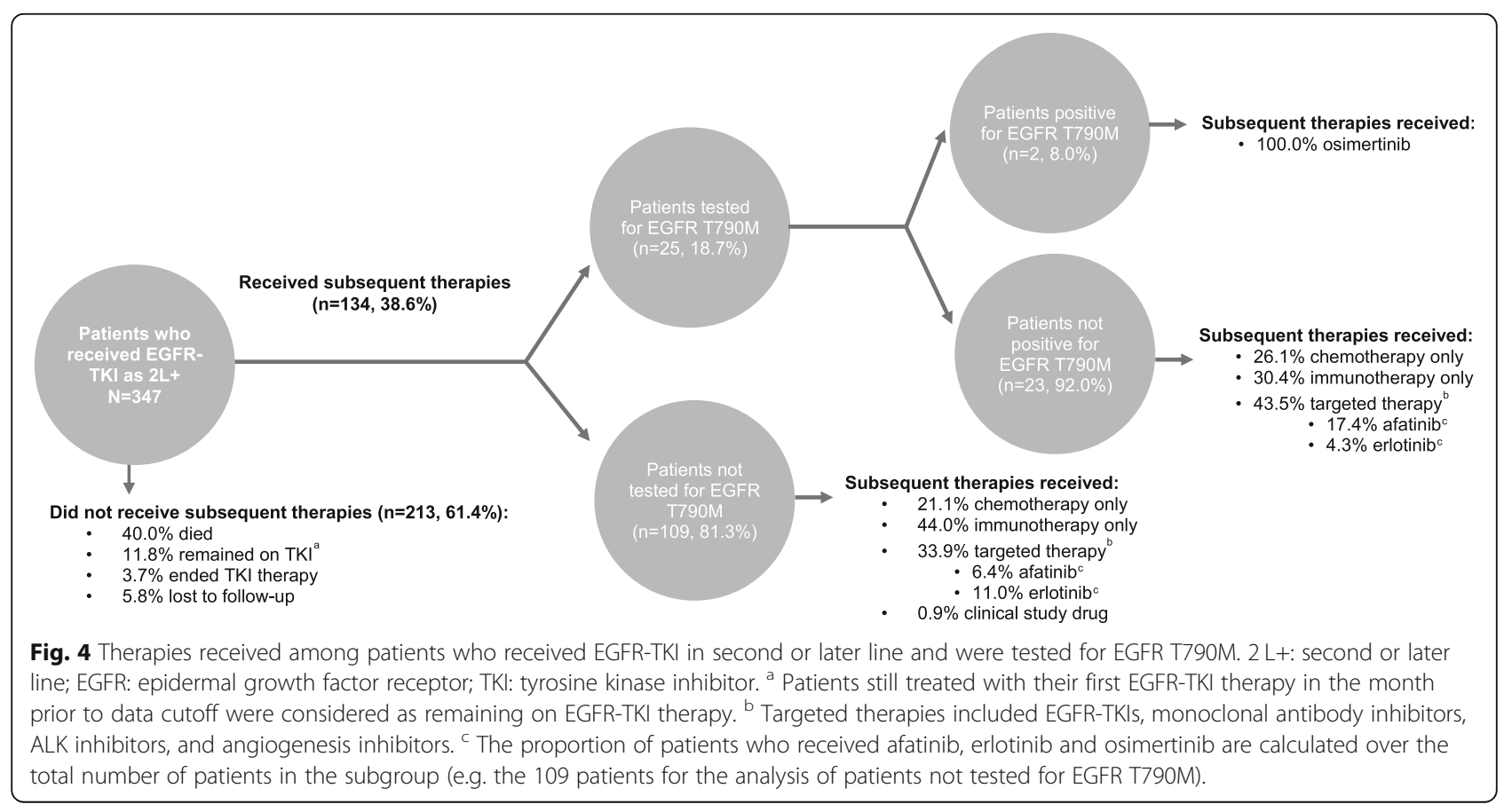


Table 5 EGFR mutation testing between index date and subsequent therapy

\begin{tabular}{|c|c|c|c|}
\hline \multirow[t]{2}{*}{ Types of Biomarkers } & $\begin{array}{l}\text { Patients with } \\
\text { subsequent therapy }\end{array}$ & $\begin{array}{l}\text { Patients who received } \\
1 \mathrm{~L} \text { EGFR-TKI and had } \\
\text { subsequent therapy }\end{array}$ & $\begin{array}{l}\text { Patients who received } \\
2 \mathrm{~L}+\mathrm{EGFR}-\mathrm{TKI} \text { and had } \\
\text { subsequent therapy }\end{array}$ \\
\hline & $\begin{array}{l}(\boldsymbol{N}=294) \\
n(\%)\end{array}$ & $\begin{array}{l}(\boldsymbol{N}=160) \\
n(\%)\end{array}$ & $\begin{array}{l}(\boldsymbol{N}=134) \\
n(\%)\end{array}$ \\
\hline Tested & $88(29.9)$ & $63(39.4)$ & $25(18.7)$ \\
\hline Positive $^{a}$ & $47(53.4)$ & $39(61.9)$ & $8(32.0)$ \\
\hline T790M mutation & $25(53.2)$ & $23(59.0)$ & $2(25.0)$ \\
\hline Exon 19 deletion & $21(44.7)$ & $16(41.0)$ & $5(62.5)$ \\
\hline L858R point mutation in exon 21 & $11(23.4)$ & $9(23.1)$ & $2(25.0)$ \\
\hline Other mutation type & $2(4.3)$ & $1(2.6)$ & $1(12.5)$ \\
\hline Unknown & $0(0.0)$ & $0(0.0)$ & $0(0.0)$ \\
\hline Negative & $38(43.2)$ & $22(34.9)$ & $16(64.0)$ \\
\hline Results pending & $2(2.3)$ & $1(1.6)$ & $1(4.0)$ \\
\hline Unsuccessful / indeterminate test & $1(1.1)$ & $1(1.6)$ & $0(0.0)$ \\
\hline \multicolumn{4}{|l|}{ Sample } \\
\hline Tissue & $48(54.5)$ & $36(57.1)$ & $12(48.0)$ \\
\hline Blood & $35(39.8)$ & $24(38.1)$ & $11(44.0)$ \\
\hline Unknown & $3(3.4)$ & $2(3.2)$ & $1(4.0)$ \\
\hline Urine & $2(2.3)$ & $1(1.6)$ & $1(4.0)$ \\
\hline
\end{tabular}

$1 L$ first line, $2 L+$ second or later line, EGFR epidermal growth factor receptor, EGFR-TKI epidermal growth factor receptor-tyrosine kinase inhibitor

${ }^{a}$ EGFR mutations are reported among those patients with a positive EGFR test result. Patients may have more than one EGFR mutation

and test reimbursements, which may affect adherence to testing guidelines during the treatment process for patients with NSCLC [24]. Lastly, other barriers in all settings include inability to wait for testing and delay start of treatment, tissue insufficiency, potential harm from repeat biopsies, poor patient health and inability to undergo testing $[24,25]$. Of note, $12.5 \%$ of included patients had SCC, and these patients are less likely to have undergone testing due to the low frequency of EGFR mutations and differences in clinical guidelines [6-8].

Our study reported median time from metastatic NSCLC diagnosis to EGFR-TKI initiation in $1 \mathrm{~L}$ of 62 days. A recent scoping review reported mean/median time from NSCLC diagnosis to treatment ranging from 15 to 60 days in the US [26]. There are limited studies evaluating time from metastatic NSCLC diagnosis to treatment; however, one study reported $29 \%$ of patients waited more than 90 days from initial presentation to clinician to treatment, of whom $49 \%$ were stage IIIA-IV [27]. Prior studies have suggested challenges to achieving shorter time to treatment, including delayed return of results and lack of communication of positive findings to the clinician and/or patient, while some are administrative including changes in insurance for patients [26, 27]. This underscores the need for broad-based education throughout the oncology community, plus further research to understand EGFR mutation testing uptake and barriers to accessing current state technologies.
Since 2013, clinical guidelines recommend repeat molecular testing upon progression on first- and secondgeneration EGFR-TKIs to detect mutations such as T790M [7]. Prior to 2015, there were no standard therapeutic options for patients with metastatic NSCLC with acquired resistance to initial EGFR-TKI [28]. Osimertinib has led to a paradigm shift in the management of patients with metastatic NSCLC who develop T790M and experience disease progression on first- or secondgeneration EGFR-TKIs. The need for tumor genotyping is thus highly supported at least at time of diagnosis and disease progression on or after first- or second-generation EGFR-TKIs for mutation evaluation. Nevertheless, only approximately $30 \%$ of patients in this study who received first- or second-generation EGFR-TKIs and progressed were tested for T790M prior to initiating subsequent therapy. The proportion of patients tested for EGFR mutations on progression prior to initiating subsequent therapy was lower in patients treated with an EGFR-TKI in $1 \mathrm{~L}$ versus $2 \mathrm{~L}+$ (39\% versus 19\%). Among patients tested for T790M, $28 \%$ were positive and mostly received osimertinib. One rationale for the lower proportion of patients tested for EGFR mutations on progression may be that EGFR testing was not recommended prior to FDA approval of osimertinib [6-8]; therefore, EGFR testing following progression was slowly being adopted during this study. In any case, $30 \%$ of patients who received first- or second-generation EGFR-TKI died before receiving any subsequent therapy 
and only $38 \%$ of patients treated with EGFR-TKI had subsequent therapy. Another recent study reported that 30\% of patients with EGFR mutations were not treated with subsequent therapy due to fast disease progression and death [29].

In previous studies among patients with NSCLC who progressed on $1 \mathrm{~L}$ EGFR-TKI therapy, approximately $50 \%$ were $\mathrm{T} 790 \mathrm{M}$ positive $[10,11]$. Our study indicates a lower proportion of $\mathrm{T} 790 \mathrm{M}$ positive patients $(1 \mathrm{~L}=37 \%$, $2 \mathrm{~L}+=8 \%$ ). This lower rate is at least partially due to the fact that almost half of patients included in our study lacked evidence of EGFR-TKI sensitizing mutations at baseline. Lower rates of T790M detection may also be attributed to use of plasma/serum (blood) for EGFR testing, and sensitivities of laboratory techniques in the detection of the mutation [30, 31]. Recent prospective studies showed higher T790M detection rates in tissue versus plasma samples [30, 32]. In our study, either tissue $(1 \mathrm{~L}=57 \% ; 2 \mathrm{~L}+=38 \%)$ or blood $(1 \mathrm{~L}=48 \% ; 2 \mathrm{~L}+=44 \%)$ was used for EGFR testing upon progression. Depending on the laboratory technique used to detect T790M, sensitivities can range from as low as $29-82 \%$, and specificities from 83 to $100 \%$ [31]. Laboratory techniques with lower sensitivities may not accurately identify T790M. The database does not detail methods of detection (e.g. Cobas [Roche], ddPCR [Bio-Rad]) used to identify T790M; therefore, we cannot be certain that observed lower T790M detection rates are attributable to detection methods associated with low sensitivities.

In April 2018, osimertinib received approval as $1 \mathrm{~L}$ treatment in patients with EGFR ex19del/L858R-positive metastatic NSCLC [33]. The approval was based on the FLAURA trial which demonstrated that, in previouslyuntreated EGFR mutation-positive NSCLC, median progression-free survival (osimertinib $=18.9$ months, comparator $=10.2$ months, hazard ratio $=0.46)$ and overall survival (osimertinib $=38.6$ months, comparator $=$ 31.8 months, hazard ratio $=0.80$ ) were longer among patients receiving osimertinib compared with standard EGFR-TKIs $[4,16]$. More recently, a global multicenter retrospective (GioTag) study demonstrated a median time on chemotherapy-free treatment of 27.6 months among patients with metastatic NSCLC with EGFRmutation (ex19del/L858R) who received $1 \mathrm{~L}$ afatinib followed by second-line osimertinib [34]. Although there was clinical benefit in sequential treatment of afatinib followed by osimertinib, findings from the current study show that $30 \%$ of the patients who received first- or second-generation EGFR-TKI died before receiving any subsequent therapy, and only $30 \%$ were tested for T790M upon progression, resulting in a small number of patients $(47[16 \%])$ actually receiving osimertinib as a subsequent therapy option. Consequently, many patients with metastatic NSCLC may not have survived long enough to be EGFR-tested and treated with osimertinib as subsequent therapy. The low rate of documented EGFR mutation positivity at baseline may have contributed to this high mortality rate, since EGFR-TKI therapy may have suboptimal efficacy in this setting. Given the results from FLAURA, considering $1 \mathrm{~L}$ treatment that could maximize clinical benefit for all eligible EGFR mutation-positive patients will be an important treatment decision for patients moving forward. Since there has been evolution in the treatment landscape of EGFR mutation-positive patients with approval of $1 \mathrm{~L}$ osimertinib, future research is needed to evaluate its impact on treatment patterns, outcomes in the real-world setting, and the molecular basis of acquired resistance to osimertinib.

This study has several strengths. First, with a nationally representative dataset including both structured and unstructured data processing, the database offers a unique opportunity to study disease progression among cancer patients. Second, the large patient population was drawn primarily from community-based practices (> 85\%); results of this study are generalizable to US community-based oncology practices [35]. Third, slightly more patients who were T790M negative received immunotherapy alone $(1 \mathrm{~L}=20 \%)$ vs chemotherapy alone $(1 \mathrm{~L}=18 \%)$ as subsequent therapy, despite clinical guidelines recommending chemotherapy in the second-line setting for T790M negative patients [6]. These unique findings provide invaluable insight to real-world community practice patterns. Lastly, we evaluated testing and treatment patterns in the overall study sample, and further stratified patients who received EGFR-TKI in $1 \mathrm{~L}$ and $2 \mathrm{~L}+$, enabling assessment of characteristics of testing and treatment patterns by line of therapy.

Some limitations of the study should be noted. First, data on some clinically important patient characteristics were not available in the database. For example, ECOG performance status was available for approximately $50 \%$ of patients, therefore description of patient characteristics is based on available data. Second, the indication for erlotinib changed in late 2016 for patients with NSCLC receiving maintenance or $2 \mathrm{~L}+$ treatment, to limit use to those whose tumors have EGFR ex19del or L858R mutations [36]. Therefore, some patients in the current study who were not EGFR mutation-positive may have received erlotinib as second-line or maintenance therapy. The subset of patients who received EGFR-TKI in $1 \mathrm{~L}$ may thus be more reflective of real-world mutation testing and treatment practices. Lastly, since data are drawn from community oncology centers, results may not be representative of practice at US academic medical centers.

\section{Conclusions}

This study provides insight into mutation testing and treatment patterns in patients with metastatic NSCLC in 
real-world, US, community-based practices. Findings showed that, in the community-based setting, approximately $30 \%$ of patients were tested for T790M following treatment with first- or second-generation EGFR-TKI. Among patients who underwent EGFR-TKI resistance testing, the most common EGFR mutation was T790M. Patients were initiated on $1 \mathrm{~L}$ EGFR-TKI therapy on average 2 months after the initial diagnosis. These significant delays signal the presence of medical and administrative barriers that hinder the rapid initiation of EGFR-TKI therapy; overcoming these barriers may be key to optimize patient outcomes. Given that $30 \%$ of the patients died without receiving any further therapy and that only $30 \%$ of those who progressed were tested for T790M, this study highlights the importance of choosing the most optimal treatment option for patients with EGFR-mutated NSCLC in the $1 \mathrm{~L}$ setting.

\section{Abbreviations}

$1 \mathrm{~L}$ : first line; $2 \mathrm{~L}+$ : second or later line; EGFR: Epidermal growth factor receptor; EHR: Electronic health records; ex19del: exon 19 deletion; NSCLC: Non-small cell lung cancer; TKl: Tyrosine kinase inhibitor

\section{Acknowledgements}

Sara Kaffashian, Caroline Korves, and Nora McCormick of Analysis Group provided editorial support for this manuscript.

\section{Authors' contributions}

ACC, AWF, MP, JWW, FL, MSD, NC, JS: study conception and design. MP, FL, JS: literature research. ACC, AWF, FL, JS: experimental studies / data analysis. MP, FL, MSD: statistical analysis. AWF, MP, FL, NC: manuscript preparation. ACC, AWF, MP, JWW, FL, MSD, NC, JS: manuscript editing. All authors read and approved the final version of the manuscript.

\section{Funding}

This research was funded by AstraZeneca US (Wilmington, Delaware). The funder contributed to the conception and design of the study. The authors would like to acknowledge Natasha Cary, BSc, of iMed Comms, Macclesfield, UK, an Ashfield Company, part of UDG Healthcare plc, for technical editing support that was funded by AstraZeneca in accordance with Good Publications Practice (GPP3) guidelines (http://www.ismpp.org/gpp3).

\section{Availability of data and materials}

Data used for this study are from Flatiron Health and were used under license for the current study. Therefore, restrictions apply to the availability of these data, which are not publicly available. Access to this data set is available to other interested parties for a fee set by Flatiron Health (https:// flatiron.com/contact/). No administrative permissions were required to access the raw data.

\section{Ethics approval and consent to participate}

All procedures performed in studies involving human participants were in accordance with the ethical standards of the institutional and/or national research committee and with the 1964 Helsinki declaration and its later amendments or comparable ethical standards. The New England Independent Review Board (NEIRB) determined that this research activity was exempt from NEIRB review, under the following category:

Research involving the collection or study of existing data, documents, records, pathological specimens, or diagnostic specimens, if the investigator records the information in such a manner that subjects cannot be identified, directly or through identifiers linked to the subjects.

The Copernicus Group Institutional Review Board approved the waiver of informed consent prior to initiation of this study.

\section{Consent for publication}

Not applicable.

\section{Competing interests}

ACC has participated in advisory boards for, or received consultancy fees or research grants from AstraZeneca, AbbVie, Bristol-Myers Squibb, Genetech/ Roche, and Lilly. AWF, MP, and NC are employees of AstraZeneca. JWW, FL, and MSD are employees of Analysis Group, Inc., a consulting company that has received research funds from AstraZeneca. JS has received consulting fees, speaking fees and research grants from AstraZeneca, Biocept, Boehringer-Ingelheim, Lilly, Paradigm, and Pfizer.

\section{Author details}

${ }^{1}$ Yale School of Medicine, 20 York Street, New Haven, CT 06510, USA.

${ }^{2}$ AstraZeneca US, 950 Wind River Ln, Gaithersburg, MD 20878, USA. ${ }^{3}$ Groupe d'analyse, Ltée, Deloitte Tower, 1190 Avenue des Canadiens-de-Montréal Suite 1500, Montreal, QC H3B 0G7, Canada. ${ }^{4}$ Analysis Group, Inc., 111 Huntington Avenue, 14th Floor, Boston, MA 02199, USA. ${ }^{5}$ Saint Luke's Cancer Institute, 4321 Washington St, Medical Plaza III, Ste 4000, Kansas City, MO 64111, USA.

Received: 25 October 2019 Accepted: 5 April 2020

Published online: 28 April 2020

\section{References}

1. Mok TS, Wu YL, Thongprasert S, Yang CH, Chu DT, Saijo N, et al. Gefitinib or carboplatin-paclitaxel in pulmonary adenocarcinoma. N Engl J Med. 2009; 361(10):947-57.

2. Rosell R, Carcereny E, Gervais R, Vergnenegre A, Massuti B, Felip E, et al. Erlotinib versus standard chemotherapy as first-line treatment for European patients with advanced EGFR mutation-positive non-small-cell lung cancer (EURTAC): a multicentre, open-label, randomised phase 3 trial. Lancet Oncol. 2012;13(3):239-46.

3. Sequist LV, Yang JC, Yamamoto N, O'Byrne K, Hirsh V, Mok T, et al. Phase III study of afatinib or cisplatin plus pemetrexed in patients with metastatic lung adenocarcinoma with EGFR mutations. J Clin Oncol. 2013;31 (27):3327-34.

4. Soria JC, Ohe Y, Vansteenkiste J, Reungwetwattana T, Chewaskulyong B, Lee $\mathrm{KH}$, et al. Osimertinib in untreated EGFR-mutated advanced non-small-cell lung Cancer. N Engl J Med. 2018;378(2):113-25.

5. Costa DB, Halmos B, Kumar A, Schumer ST, Huberman MS, Boggon TJ, et al. BIM mediates EGFR tyrosine kinase inhibitor-induced apoptosis in lung cancers with oncogenic EGFR mutations. PLoS Med. 2007;4(10):1669-79 discussion 80 .

6. Hanna N, Johnson D, Temin S, Baker S Jr, Brahmer J, Ellis PM, et al. Systemic therapy for stage IV non-small-cell lung Cancer: American Society of Clinical Oncology clinical practice guideline update. J Clin Oncol. 2017;35(30):3484515.

7. Lindeman NI, Cagle PT, Beasley MB, Chitale DA, Dacic S, Giaccone G, et al. Molecular testing guideline for selection of lung cancer patients for EGFR and ALK tyrosine kinase inhibitors: guideline from the College of American Pathologists, International Association for the Study of Lung Cancer, and Association for Molecular Pathology. J Thorac Oncol. 2013;8(7):823-59.

8. Planchard D, Popat S, Kerr K, Novello S, Smit EF, Faivre-Finn C, et al. Metastatic non-small cell lung cancer: ESMO Clinical Practice Guidelines for diagnosis, treatment and follow-up. Ann Oncol. 2018;29(Supplement_4): iv192-237.

9. Ma C, Wei S, Song Y. T790M and acquired resistance of EGFR TKI: a literature review of clinical reports. J Thorac Dis. 2011;3(1):10-8.

10. Oxnard GR, Arcila ME, Sima CS, Riely GJ, Chmielecki J, Kris MG, et al. Acquired resistance to EGFR tyrosine kinase inhibitors in EGFR-mutant lung cancer: distinct natural history of patients with tumors harboring the T790M mutation. Clin Cancer Res. 2011;17(6):1616-22.

11. Yu HA, Arcila ME, Rekhtman N, Sima CS, Zakowski MF, Pao W, et al. Analysis of tumor specimens at the time of acquired resistance to EGFR-TKI therapy in 155 patients with EGFR-mutant lung cancers. Clin Cancer Res. 2013;19(8): 2240-7.

12. Cross DA, Ashton SE, Ghiorghiu S, Eberlein C, Nebhan CA, Spitzler PJ, et al. AZD9291, an irreversible EGFR TKI, overcomes T790M-mediated resistance to EGFR inhibitors in lung cancer. Cancer Discov. 2014;4(9):1046-61.

13. Yun $\mathrm{CH}$, Mengwasser KE, Toms AV, Woo MS, Greulich H, Wong KK, et al. The T790M mutation in EGFR kinase causes drug resistance by increasing the affinity for ATP. Proc Natl Acad Sci U S A. 2008;105(6):2070-5.

14. Greig SL. Osimertinib: first global approval. Drugs. 2016;76(2):263-73. 
15. US Food \& Drug Administration. FDA Approves Osimertinib for First-Line Treatment of Metastatic NSCLC With Most Common EGFR Mutations (Press release, 19 April 2018). 2018. https://www.fda.gov/drugs/resourcesinformation-approved-drugs/fda-approves-osimertinib-first-line-treatmentmetastatic-nsclc-most-common-egfr-mutations. Accessed 29 April 2019.

16. Ramalingam SS, Vansteenkiste J, Planchard D, Cho BC, Gray JE, Ohe Y, et al. Overall survival with Osimertinib in untreated, EGFR-mutated advanced NSCLC. N Engl J Med. 2020;382(1):41-50.

17. Lindeman NI, Cagle PT, Aisner DL, Arcila ME, Beasley MB, Bernicker EH, et al. Updated molecular testing guideline for the selection of lung Cancer patients for treatment with targeted tyrosine kinase inhibitors: guideline from the College of American Pathologists, the International Association for the Study of Lung Cancer, and the Association for Molecular Pathology. J Thorac Oncol. 2018;13(3):323-58.

18. Flatiron: Flatiron Health database. September 2017. 2017.

19. Flatiron: Flatiron Health database, mortality v2.0. September 2017. 2017.

20. Lee DH, Tsao MS, Kambartel KO, Isobe H, Huang MS, Barrios CH, et al. Molecular testing and treatment patterns for patients with advanced non-small cell lung cancer: PlvOTAL observational study. PLoS One. 2018;13(8):e0202865.

21. Chouaid C, Dujon C, Do P, Monnet I, Madroszyk A, Le Caer H, et al. Feasibility and clinical impact of re-biopsy in advanced non small-cell lung cancer: a prospective multicenter study in a real-world setting (GFPC study 12-01). Lung Cancer. 2014;86(2):170-3.

22. Hong MH, Kim HR, Ahn BC, Heo SJ, Kim JH, Cho BC. Real-world analysis of the efficacy of Rebiopsy and EGFR mutation test of tissue and plasma samples in drug-resistant non-small cell lung Cancer. Yonsei Med J. 2019; 60(6):525-34.

23. Kim TO, Oh IJ, Kho BG, Park HY, Chang JS, Park CK, et al. Feasibility of rebiopsy and EGFR mutation analysis in patients with non-small cell lung cancer. Thorac Cancer. 2018;9(7):856-64.

24. Gutierrez ME, Choi K, Lanman RB, Licitra EJ, Skrzypczak SM, Pe Benito R, et al. Genomic profiling of advanced non-small cell lung Cancer in community settings: gaps and opportunities. Clin Lung Cancer. 2017; 18(6):651-9.

25. Lim C, Tsao MS, Le LW, Shepherd FA, Feld R, Burkes RL, et al. Biomarker testing and time to treatment decision in patients with advanced nonsmallcell lung cancer. Ann Oncol. 2015;26(7):1415-21.

26. Malalasekera A, Nahm S, Blinman PL, Kao SC, Dhillon HM, Vardy JL. How long is too long? A scoping review of health system delays in lung cancer. Eur Respir Rev. 2018;27(149):180045.

27. Vidaver RM, Shershneva MB, Hetzel SJ, Holden TR, Campbell TC. Typical time to treatment of patients with lung Cancer in a multisite, US-Based Study. J Oncol Pract. 2016;12(6):e643-53.

28. Santarpia M, Liguori A, Karachaliou N, Gonzalez-Cao M, Daffina MG, D'Aveni $A$, et al. Osimertinib in the treatment of non-small-cell lung cancer: design, development and place in therapy. Lung Cancer (Auckl). 2017;8:109-25.

29. Roeper J, Falk M, Tiemann M, Wesseler C, Wiest G, Sackmann S, et al. Risk of not receiving 2 nd line therapy is high in EGFR $\mathrm{mt}+$ pts: Real world data of certified lung cancer centers on treatment sequence in EGFR mt+ pts. J Clin Oncol. 2018;36(15_suppl):e21220.

30. Kobayashi K, Naoki K, Manabe T, Masuzawa K, Hasegawa H, Yasuda H, et al. Comparison of detection methods of EGFR T790M mutations using plasma, serum, and tumor tissue in EGFR-TKI-resistant non-small cell lung cancer. Onco Targets Ther. 2018;11:3335-43.

31. Li X, Zhou C. Comparison of cross-platform technologies for EGFR T790M testing in patients with non-small cell lung cancer. Oncotarget. 2017;8(59): 100801-18.

32. Kanai K, Yamamoto N, Nogami N, Atagi S, Saka H, Tashiro N, et al. 141PD a prospective study of molecular testing status in the EGFR mutation positive NSCLC patients with disease progression during EGFR TKI treatment (REMEDY study). J Thorac Oncol. 2018;13(4):S82-3.

33. Food and Drug Administration (FDA). FDA approves osimertinib for first-line treatment of metastatic NSCLC with most common EGFR mutations. https://www.fda.gov/Drugs/InformationOnDrugs/ApprovedDrugs/ucm6 05113.htm. Accessed 23 May 2018.

34. Hochmair MJ, Morabito A, Hao D, Yang CT, Soo RA, Yang JC, et al. Sequential treatment with afatinib and osimertinib in patients with EGFR mutation-positive non-small-cell lung cancer: an observational study. Future Oncol. 2018;14(27):2861-74.
35. Berger ML, Curtis MD, Smith G, Harnett J, Abernethy AP. Opportunities and challenges in leveraging electronic health record data in oncology. Future Oncol. 2016;12(10):1261-74.

36. FDA. Tarceva (erlotinib) prescribing information. 2016. https://www. accessdata.fda.gov/drugsatfda_docs/label/2016/021743s025lbl.pdf. Accessed 11 October 2019

\section{Publisher's Note}

Springer Nature remains neutral with regard to jurisdictional claims in published maps and institutional affiliations.
Ready to submit your research? Choose BMC and benefit from:

- fast, convenient online submission

- thorough peer review by experienced researchers in your field

- rapid publication on acceptance

- support for research data, including large and complex data types

- gold Open Access which fosters wider collaboration and increased citations

- maximum visibility for your research: over $100 \mathrm{M}$ website views per year

At BMC, research is always in progress.

Learn more biomedcentral.com/submissions 